\title{
Pâturages
}

\section{Le sens de La conduite}

La transhumance de noé de Barras en 1480

\section{William Blanc}

\section{(2) OpenEdition}

Journals

Édition électronique

URL : https://journals.openedition.org/tc/7527

DOI : $10.4000 /$ tc. 7527

ISBN : 0248-6016

ISSN : 1952-420X

Éditeur

Éditions de l'EHESS

\section{Édition imprimée}

Date de publication : 1 mai 2015

Pagination : 282-289

ISBN : 9782713224874

ISSN : 0248-6016

Référence électronique

William Blanc, «Le sens de La conduite », Techniques \& Culture [En ligne], 63 | 2015, mis en ligne le 16 décembre 2018, consulté le 29 septembre 2022. URL : http://journals.openedition.org/tc/7527 ; DOI : https://doi.org/10.4000/tc.7527 


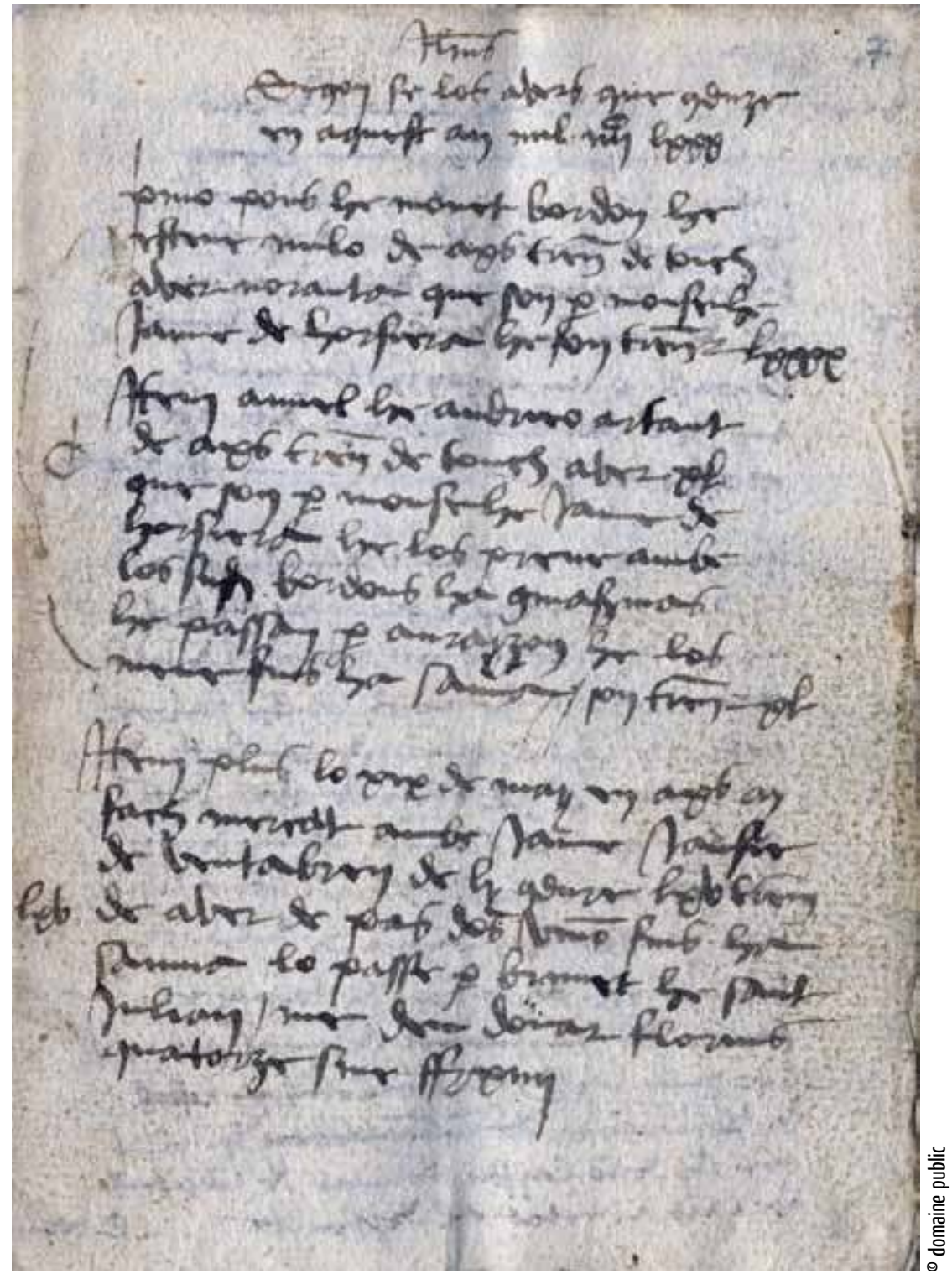




\section{LE SENS DE LA CONDUITE}

\section{La transhumance de Noé de Barras en 1480}

Nous connaissons la transhumance organisée par Noé de Barras à travers son livre de compte dédié presque exclusivement à cette activité pour l'année 1480. En l'étudiant, nous pouvons comprendre comment s'organisait ce flux économique complexe qui mettait en jeux des capitaux importants. Outre les bergers, les propriétaires de troupeaux, les loueurs d'estives et les tenants de péages (les passages), apparaît un personnage intermédiaire, le conducteur, dont le rôle est double. Il garantit d'abord aux bergers et aux propriétaires une véritable assurance contre d'éventuels abus aux passages, tenus soit par des seigneurs locaux, soit par des communautés de moyenne montagne. Du côté de ces dernières, il sert de garant, notamment en cas de dégâts causés aux cultures par les troupeaux. Cette place d'intermédiaire, Noé de Barras l'assume grâce à son appartenance à une famille de noblesse ancienne dont les fiefs (et les fiefs de ses collatéraux) s'étalent sur le chemin de la transhumance. Il vient aussi de se marier, en 1479, à une proche du plus important loueur d'estives du Haut Dauphiné, Jacques d'Orcières. Il occupe enfin une fonction de magistrat local.

Cette position centrale sociale et géographique place Noé de Barras au milieu d'un réseau dont il monopolise les informations, concentrées dans son livre, qui lui assurent des revenus importants. Mais il ne s'agit pas de la seule raison poussant un noble à s'engager dans une pareille activité. Le bénéfice symbolique est lui aussi important.

Les figures du berger et de l'agneau dans la société chrétienne féodale renvoient à un ensemble de références religieuses importantes. Le berger, c'est le Christ, c'est le clerc, mais c'est aussi, à partir du début du xvI ${ }^{\mathrm{e}}$ siècle, le roi (Beaune 2009). Dans certaines cérémonies princières, comme en 1389 à Mazère - ville proche des Pyrénées et des terres de transhumance -, Charles VI est accueilli par des chevaliers grimés en bergers conduisant 


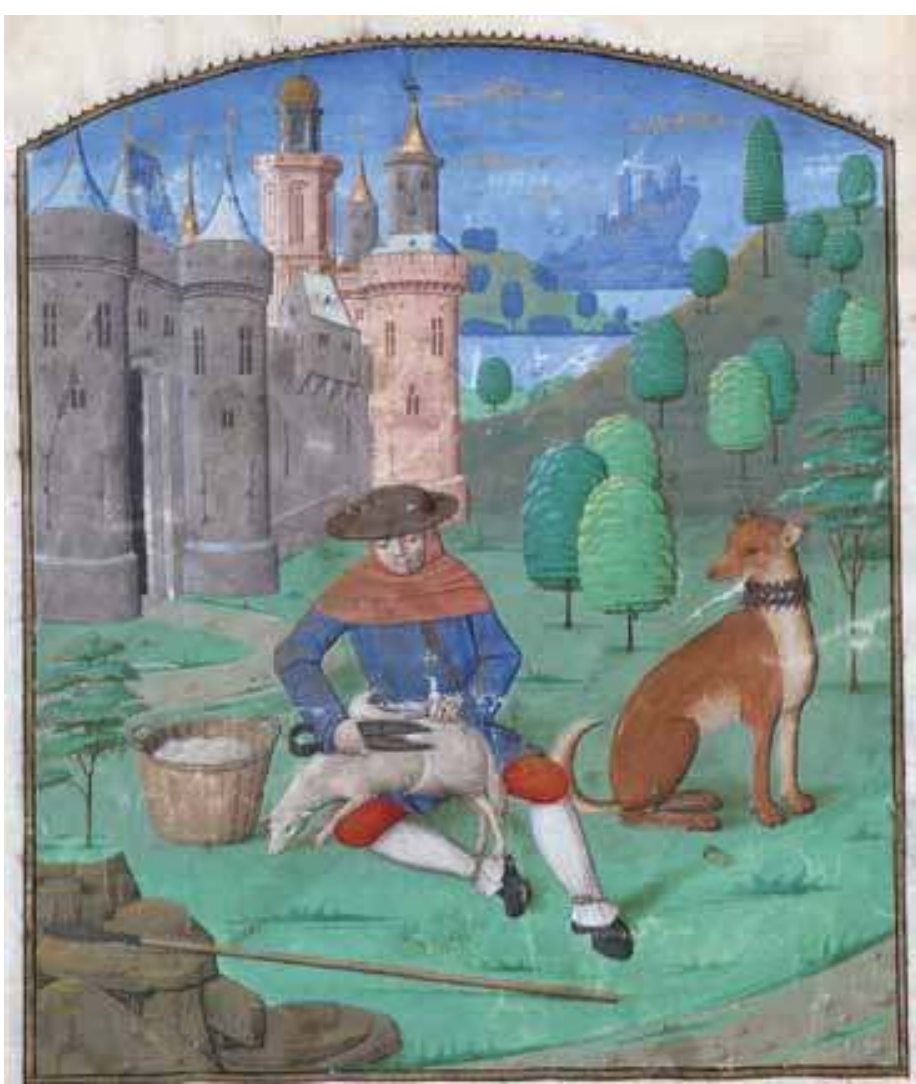

Dans ce manuscrit offert à Louis $\mathrm{XI}$ au crépuscule de sa vie, le roi est représenté comme un berger qui sait tondre à raison ses bêtes. Dans cette métaphore, les moutons représentent le peuple qu'un roi sage se doit de ne pas imposer trop durement (IMAGE 2). Le souverain est aussi celui qui doit veiller à la prospérité du troupeau et que ses chiens (ici la noblesse de cour, les magistrats, l'armée) remplissent leur fonction. À défaut, les ovins seront menacés et, avec eux, tout le vaisseau de l'État. Comme l'explique le quatrain sous l'enluminure de l'image 3: « Se mon chien eust bien veillé sur le loup, jamais il n'eust ma brebis estranglée. Or l'ay tué maintenant de ung seul coup, mais non pourtant ma brebis est finee [morte]. » (Choinet, Scordia, 2009). Depuis Charles VI, des scènes pastorales sont incluses dans les entrées royales.
Choinet P. (auteur),

Maître de l'échevinage

(enlumineur), 1482-1483, Le

livre des trois âges, BNF, mss.

Smith Lesouef 70 , folio 9

verso.

Choinet P. (auteur),

Maître de l'échevinage

(enlumineur), 1482-1483, Le

livre des trois âges, BNF, mss.

Smith Lesouef 70 , folio 11

verso.

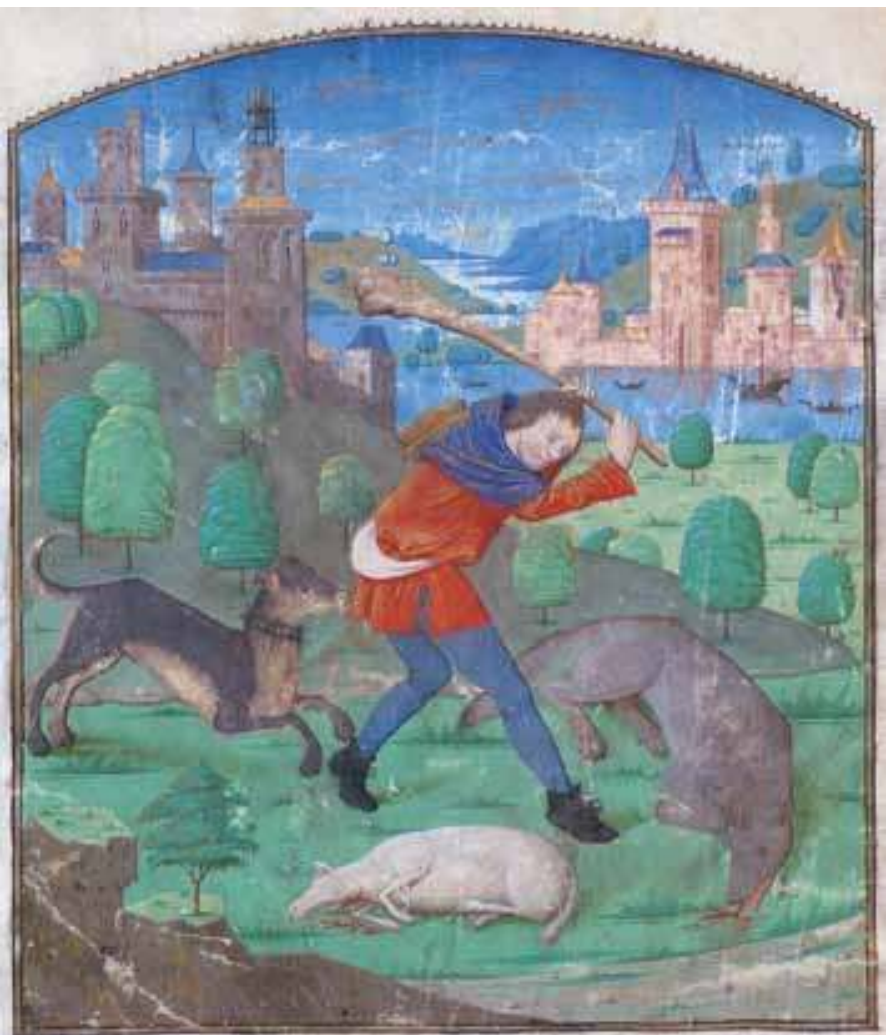


des moutons ${ }^{1}$. Presque un siècle plus tard, dans Le livre des trois âges offert à Louis XI, le roi est représenté comme un berger. Or, une pareille analogie fait sens, tout comme l'origine légendaire de la possession des bovins justifie et distingue les peuples, les genres, et les fonctions sociales dans l'« univers du bétail » de l'Afrique subsaharienne étudié par Pierre Bonte (2004).

En effet, dans la société médiévale, les animaux ne sont pas qu'un symbole. Ils exercent aussi une fonction importante dans le processus d'affirmation du dominium, qui, en l'absence du concept moderne de propriété, représente l'étendue du pouvoir - et pas des droits qu'exerce un seigneur sur une terre ou sur des hommes et qu'il doit, sans l'aide d'outils écrits, régulièrement faire reconnaître à travers des rites sociaux - comme l'hommage -. La chasse, comme l'a noté Joseph Morsel, notamment celle du cerf à partir des XI ${ }^{e}$-XII ${ }^{e}$ siècle, est ainsi utilisée au xII par l'abbé Suger pour établir son dominium sur la forêt d'Yveline (Morsel, 2002) tout en redistribuant le gibier tué aux pauvres et à ses hommes qui, en le consommant, pourrait-on dire, mangent de l'espace social. Les troupeaux d'élevage peuvent occuper la même fonction (Morsel ,2004). On a ainsi noté que les grandes transhumances ibériques avaient été une arme de la Reconquista de la Mancha et de l'Estremadura aux $\mathrm{XII}^{\mathrm{e}}$-XIII ${ }^{e}$ siècles ${ }^{2}$, pratique transposée au nord du Mexique par les rancheros à partir du XvII $^{e}$ siècle (Bischko, 1963). Cette prise en main directe peut aussi être indirecte. En Italie, au Xve siècle, la Douane de la Chambre apostolique (l'administration papale) accordait une protection aux éleveurs venant hiverner dans des pâturages lui appartenant (dans le Patrimonio), contre une redevance appelée la fida. En la payant, les bergers entraient dans un rapport féodal avec la Chambre et devenaient, le temps d'une transhumance, ses fidati (fidèles), statut qui garantissait non seulement leur personne, mais aussi leurs biens (Maire-Vigueur, 1981). Le déplacement des hommes et bêtes permettait ainsi une réactivation saisonnière du dominium de la Chambre Apostolique sur le Patrimonio. En Provence, le procédé est similaire et s'étend même sur des terres où, suite à la crise du xiv ${ }^{e}$ siècle, la famille de Noé de Barras a dû céder ou partager ses fiefs. Venant réparer des dommages commis par les troupeaux qu'il a à sa charge, le conducteur agit, sur des territoires qui ne sont pas à lui, comme un seigneur justicier.

Dans son livre, Noé de Barras affirme qu'il « conduit» des troupeaux. Le terme de « conducteur » apparaît lui sous la plume d'un des tenants de passage qui le désigne sous la quasi-titulature de «noblilis Noe de Barrassio, conductor averium provincialium » ( le noble Noé de Baras, « conducteur » des troupeaux provençaux »). Que signifie ce terme et sa famille lexicale au Moyen âge? Dans Le chevalier à la Charrette de Chrétien de Troyes (fin du XII ${ }^{e}$ siècle), le conduit désigne la protection accordée par le roi: « Vos feites mal, seignor, car li rois nos conduit, nos somes an sa garde tuit. ${ }^{3} \mathrm{Au} \mathrm{Xv}^{e}$ siècle, dans une ordonnance de Louis XI, le verbe conduire peut être synonyme de gouverner comme dans l'ordonnance royale du 27 mai 1474: « donnons, par cesdictes presentes, plain pouvoir, authorité, commission et mandement especial, de traicter, conduire et gouverner les affaires communes de ladicte ville de Bourges » (Pastoret, 1828). Le substantif « conducteur » désigne également des fonctions militaires en France, et ce dès la fin du XIV siècle dans La Chanson de Bertrand du Guesclin où il est dit, vers 18045 et 18046 que le connétable « Retint et soudoia, et paia sans errour IIIIM [4000] combattans dont il fu conductour. » C'est également le cas dans l'armée bourguignonne où, d'après l'ordonnance de 1473 de Saint-Maximin-de-Trèves, conducteur renvoyait à l'un des grades les plus élevé des troupes ducales et désignait l'homme, issu des rangs de la noblesse, qui dirigeait une compagnie d'ordonnance de près de neuf cents combattants (Schnerb, 2005). Pensons enfin aux condottieres de la péninsule italienne. 


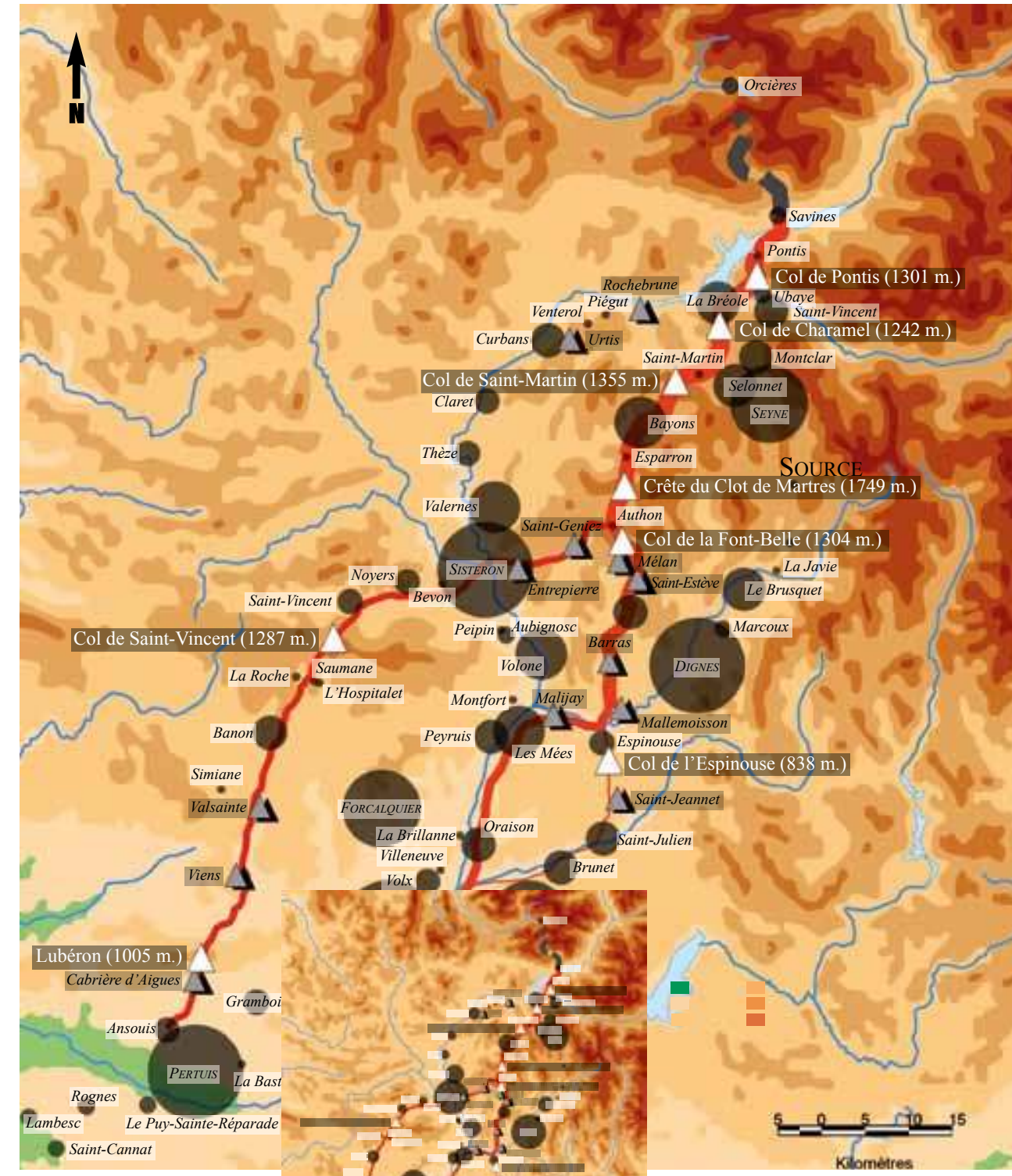

LÉGENDE

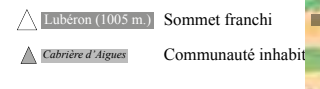

Altimétrie

$100-200 \mathrm{~m} \quad 1000-1500 \mathrm{~m}$

$200-500 \mathrm{~m} \quad 1500-2000 \mathrm{~m}$

$500-1000 \mathrm{~m} \square 2000-2500 \mathrm{~m}$

Taille de la transhumance, hypothèse haute

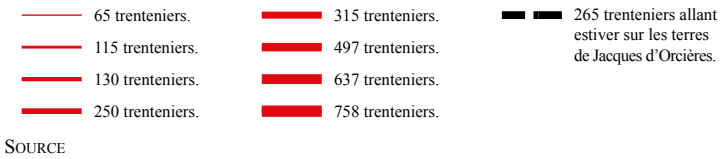

- Baratier Edouard, La démographie provençale du XIII au XVI siècle, Paris, 1961.

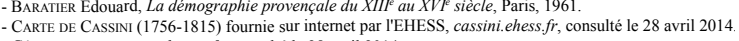

- GÉOPORTAL, geoportail.gouv. fr, consulté le 28 avril 2014

- HéBERT Michel, Regeste des états de Provence, 1347-1480, Paris, 2007. 
Lemploi du terme de conducteur pour désigner celui qui organise la transhumance, que l'on retrouve aussi dans la Dauphiné dans la famille des Claret (Verdier, 2009) ou dans l'arrêt du Parlement du Dauphiné du 2 juin 1458, qui distingue nettement cette fonction de celle des bergers ${ }^{4}$, n'a donc rien de fortuit. Il associe les grands déplacements ovins à une activité de commandement militaire et semble se développer au moment où les nobles s'investissent dans l'activité et que les princes se figurent en bergers. Il se perpétue dans le langage. Olivier de Serres (1539-1619) emprunte ainsi presque exclusivement au champ lexical militaire pour décrire la transhumance:

De l'ordonnance des Anciens \& coustumes inveterées, les mesnagers d'aujourd'hui habitans en païs importuné des chaleurs de l'esté, en telle saison envoient aux prochaines montaignes leur bestail à laine, pour y séjourner environ trois mois [...]. De plusieurs grands trouppeaux ramassés des voisins se compose une armée de bestail lanu, laquelle on baille à un général, qui aiant sous soi plusieurs capitaines, à chacun ordonne la charge d'un quartier pour tous ensemble conduire, faire paistre et coucher seurement le bestail; à ce aidés par la soigneuse garde des chiens. [... ] À cause du grand nombre de bestail séjournant à la montaigne ne peut-on s'y servir de parcs, pour enfermer le bestail durant la nuict [...] mais le couche-on parmi les terres labourées, le ramassant [...] à la manière d'une armée qui campe: ainsi le bestail environné de pasteur, de chiens et d'asnes du bagage, vivres et habits, ressemble proprement à une castramétation $^{5}$. (De Serres 1600).

On remarque le même emploi de la métaphore militaire pour décrire des troupeaux transhumant chez Cervantès, et enfin, jusqu'au début du xx ${ }^{e}$ siècle, dans plusieurs livres de Jean Grono, comme Le grand troupeau (1932).

Ces analogies ne peuvent s'expliquer par de simples licences poétiques. De Serres, Cervantès, Giono, ont tous trois vécu dans des pays habitués à l'activité transhumante. Pareillement, ce discours ne se justifie pas par la seule nécessité d'organiser les troupeaux contre les attaques de loups. Ces dernières, surtout celles dirigées contre les hommes, étaient tout aussi fréquentes en plaine qu'en montagne (Moriceau, 2007). Or, le verbe « conduire » est pratiquement absent (et dépourvu de tout sens de commandement) dans Le bon berger de Jean de Brie offert à Charles V en 1379, qui traite principalement d'élevage sédentaire en plaine et a été écrit, notons-le, non par un noble mais par un maître berger ${ }^{6}$.

Le terme de conducteur renvoie donc à une fonction de commandement aristocratique. Noé de Barras conduit un troupeau comme d'autres conduisent des armés. Ce faisant, son devoir n'est-il pas de maîtriser les dizaines de milliers de têtes transhumantes. La capacité de contrôler les animaux a été, au Moyen âge, un signe de distinction. Les saints domptent les dragons (Le Goff, 1970), les rois et les héros de chansons les chevaux fabuleux, comme Bucéphale ou Bayard. Dès le xiII ${ }^{\mathrm{e}}$ siècle, des textes littéraires font naturellement le parallèle entre la capacité à gouverner et la maîtrise de sa monture, comme dans le Lancelot-Graal où il est dit : " de même qu'on point le cheval, et le mène celui qui est dessus assis, là où il veut, de même doit le chevalier mener le peuple à son vouloir » (Roche, 2011). Ne peut-il en être pour les moutons? Cela expliquerait entre autres pourquoi, par un jeu de justifications et de discours idéels, les éleveurs de cet été 1480 aient fait appel à un jeune noble, Noé de Barras pour conduire leurs troupeaux dans un périple de deux cents kilomètres à travers la Haute Provence. 


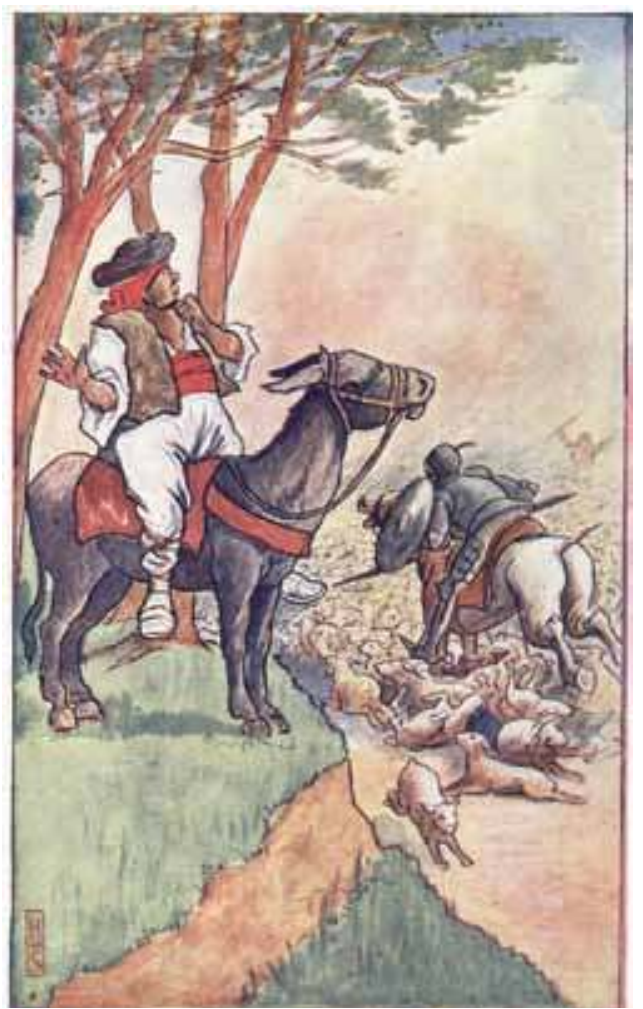

Illustration d'ouvrage

De Cervantes, M. Robarth,

E. (trad.) 1910, Don

Quixote, London, Ward: 33.
"Vois-tu cette poussière qui se lève là, Sancho? Or sache quélle est tout enflée d'une très grande armée de diverses et innombrables nations. [...] Or, la poussière qu'il avait vue, ceetaient deux grands troupeaux de brebis et de moutons, qui venaient par ce chemin-là de deux différents côtés, qui la soulevaient ainsi, et même, à cause de cette poussière, ils ne se purent apercevoir jusqu'à ce qu'ils fussent fort près. Don Quichotte affirmait avec tant de force que c'étaient des armées que Sancho en vint à le croire. » (De Cervantés, 1988). C'est ainsi que Don Quichotte décrit deux troupeaux d'ovins s'approchant de lui. L'affaire se termine mal. l'hidalgo de la Manche chargea les bêtes et en tua plusieurs, au grand dame des bergers. On remarquera qu'ici, l'aristocrate, représenté par Don Quichotte, impose à Sancho sa vision, au point que celui-ci « en vint à le croire ». Doit-on y voir une manière détourner de se moquer de la prétention de certains nobles d'assimiler la direction de la transhumance à une tâche noble et militaire au point d'en justifier leur position sociale auprès des paysans? L'épisode sera maintes fois illustré, y compris dans des pays ne connaissant pas de grandes transhumances, comme cette édition danoise du xvII" siècle, où cette édition anglaise du début du xxe siècle.

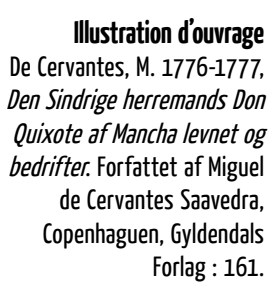

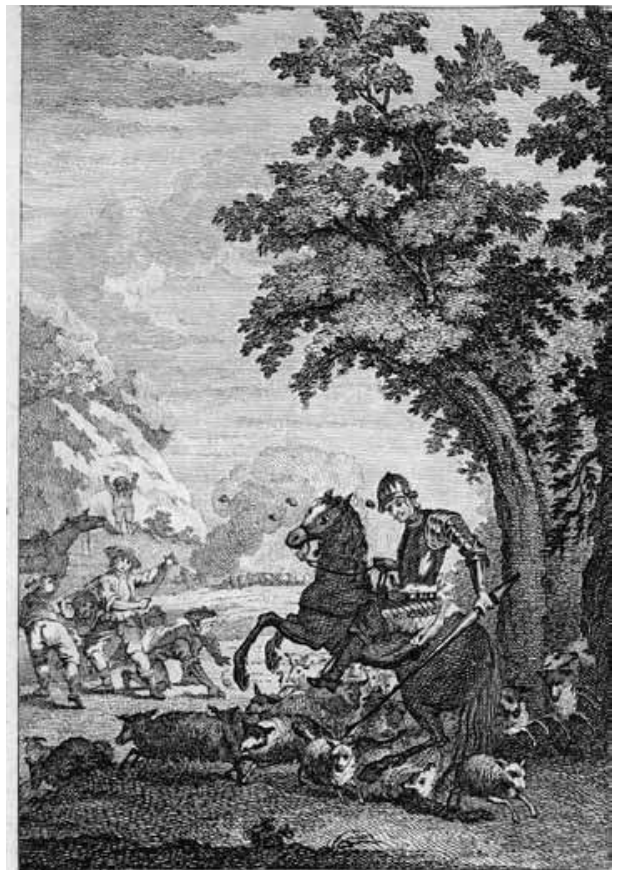

\section{NOTES}

Photo d'ouverture : Page 7 du livre de transhumance de Noé de Barras (Archives Départementales des Bouches-duRhône, cote $1 \mathrm{~J}$ 612) listant les premiers troupeaux qu'il conduit « Segon se los avers que conduze en aquest an mil CCCC LXXX » ("S'ensuivent les troupeaux que je conduis en cette année 1480 »). Véritable hapax documentaire, découvert, traduit puis édité récemment par Jean-Yves Royer (1988), le livre est un outil de la pratique économique et contient des informations que seul le conducteur possède. Les troupeaux montant en ordre dispersé, il est le seul à connaître le total des bêtes (environ 22740 bêtes réparties en plus d'une dizaine de troupeaux). Il peut donc en jouer pour faire baisser le tarif des passages, basé non sur un comptage des bêtes par les communautés, mais sur une simple déclaration du conducteur, en l'espèce, Noé de Barras. Qui irait mettre en doute la parole du magistrat local? De l'autre côté, il est le seul à connaître le coût réel des passages. Il peut là encore en jouer pour laisser le prix des passages tenus par des proches s'envoler (avec son accord), surcoût qu'il répercutera sur les rétributions qu'il demandera aux éleveurs. Agissant de la sorte, Noé de Barras est capable de maîtriser la redistribution de la richesse émise par la transhumance et d'en profiter pour reproduire son capital social et pécuniaire. 
1. Épisode rapporté par Jean Juvénal des Ursins (13881473), plusieurs décennies après, mais aussi par le Religieux de Saint-Denis, plus proche des événements: «Le roi demanda au comte [Gaston Phébus, comte de Foix] avec un air de satisfaction quels étaient ces gens qui venaient d'entrer: "Ce sont, dit le comte, vos humbles serviteurs qui sont prêts à exécuter vos ordres et à vous obéir en toutes choses, comme des pâtres et des bouviers à leur maître. "» (Bellaguet 1839).

2. Les troupeaux étaient accompagnés de véritables escortes armées, la rafala, appelé aussi l'esculca.

3. Vers 4134-4136. Traduction: "Vous agissez mal, Messeigneurs, car le roi nous protège, nous sommes tous mis sous sa sauvegarde. » (Chrétiens de Troyes 1994).

4. Larrêt, définissant en autre les droits de pulvérage (synonyme des passages) parle ainsi les « bergers et les conducteurs desdits troupeaux» (« pastorum sive conductorum dictorum averiorum. » De Salvaing 1731). On notera que les rédacteurs de l'arrêt ont fait la distinction entre les « bergers » et les « conducteurs».

5. La « castramétation » désigne l'« Art d'établir un camp. » (Trésor de la langue française). Le champ lexical renvoyant à des termes militaires a été surligné en gras par nos soins.

6. Charles Lenient a supposé en 1893 que derrière Jean de Brie se cachait en fait Charles V lui-même pour développer tout un discours métaphorique sur le prince comme berger de son peuple. L'hypothèse a été réfutée par Gustaf Holmér en 1967, qui a conclu que Jean de Brie était bien un maître berger (Wilmart 2005).

\section{POUR CITER CET ARTICLE}

Blanc, W., 2015 Le Sens de la conduite, Techniques \& Culture 63: 282-289.

\section{RÉFÉRENCES}

Beaune, C. 2009, Jeanne d'Arc. Paris: Perrin.

Bellaguet, M. L. (Dir.) 1839 Chronique du religieux de Saint-Denis. Paris: Crapelet.

Bonte, P. 2004 Des « Peuples du bétail », Techniques \& Culture [En ligne], 43-44 | 2004, mis en ligne le 10 mai 2006, consulté le 03 avril 2015. URL : http://tc.revues.org/1116

Choinet, P., Scordia, L. (Dir.), 2009 Le livre des trois âges. Mont-Saint-Aignan: Publications des universités de Rouen et du Havre.

De Brie, Jean. 1879 (1541) Le Bon berger ou le vray régime des bergers et bergères. Paris: I. Liseux.

De Cervantés, M., Canavaggio, J. (trad.) 1988 L’Ingénieux hidalgo Don Quichotte de la Manche, Livre I. Paris: Gallimard.

De Salvaing, D. 1731, De l'Usage des fiefs et autres droits seigneuriaux. Grenoble : A. Faure

De Serres, O. 1600 Le Théâtre d'agriculture et mésnage des champs. Paris : Jamet-Métayer

Troyes de, C. 1994 Romans. Paris: LGF (Le livre de Poche).

Le Goff, J. 1970, Culture ecclésiastique et folklorique au Moyen âge: saint Marcel de Paris et le dragon. In L. de Rosa Ricerche storiche ed economiche in memoria di Corrado Barbagallo. Naples: ESI.

Morsel, J. 2002 Chasse. In C. Gauvard, A. de Libera, \& M. Zink, Dictionnaire du Moyen Âge. Paris: PUF, 271-272.

— 2004, L’Aristocratie médiévale, Paris: A. Colin.

Pastoret, E. (Dir.) 1828, Ordonnances des rois de France de la troisième race, Dix-huitième volume. Paris: Imprimerie royale.

Roche, D. 2011 La Gloire et la puissance. Paris: Fayard.

Royer, J. -Y. 1988 Le Journal de Noé de Barras: un entrepreneur de transhumance au XVe siècle: texte provençal inédit de 1480. Mane, Alpes de Lumière.

Schnerb, B. 2005 L'État Bourguignon, 1363-1477. Paris: Perrin.

Verdier, R. 2009 Les Claret. Un destin nobiliaire. $x{ }^{e}-x{ }^{e}$ siècle. Grenoble: PUG.

Wilmart, M. 2005 L'Homme face à la mort de l'animal. Pratiques, savoirs et croyances des bergers du XIVe siècle d'après le traité de Jean de Brie (1379). In E. Doudet, La Mort écrite. Rites et rhétoriques du trépas au Moyen Âge. Paris: Presses Universitaires de Paris-Sorbonne. 